\title{
Free Vibration Bahaviour of Fiber Metal Lam- inates, Hybrid Composites, and Functionally Graded Beams using Finite Element Analysis
}

\author{
Harshan Ravishankar \\ Department of Mechanical Engineering, Amrita School of Engineering, Coimbatore, Amrita Vishwa \\ Vidyapeetham, Amrita University, India \\ Revathi Rengarajan \\ Department of Mechanical and Aerospace Engineering, The Ohio State University, Columbus, Ohio, USA
}

Kaliyannan Devarajan

Department of Mechanical Engineering, Amrita School of Engineering, Coimbatore, Amrita Vishwa Vidyapeetham, Amrita University, India

Bharath Kaimal
Department of Automotive Systems Engineering, The University of Michigan-Dearborn, Dearborn, USA

(Received 25 August 2014; accepted 16 February 2016)

In this study, the free vibration analysis of rotating and non-rotating fiber metal laminate (FML) beams, hybrid composite beams (HCB), and functionally graded beams (FGB) are investigated. FML beams are high-performance hybrid structures based on alternating stacked arrangements of fiber-reinforced plastic (FRP) plies and metal alloy layers. Hybrid composite beams are materials that are made by adding two different fibers. Functionally graded beams are new materials that are designed to achieve a functional performance with gradually variable properties in one or more directions. The effects of different metal alloys, composite fibers, and different aspect ratios and angular velocities on the free vibration analysis of FML beams are studied. The effects of different angular velocities and different aspect ratios of rotating and non-rotating hybrid composite beams are also investigated. Finally, the effects of different angular velocities and different material distributions, namely the power law, exponential distribution, and Mori Tanaka's scheme on the free vibration analysis of FGB, are also invesigated.

\section{INTRODUCTION}

Fiber metal laminates, hybrid composites, and functionally gradient beams are often used in engineering applications. Rotating FML, HCB, and FGB are especially used in helicopter and wind turbine blades. FML are hybrid structures consisting of different metal sheets and FRP composite layers such as glass, aramid, and carbon fibers. One of the most important objectives of their production is to combine the good impact resistance of the metals with the light weight characteristic of FRP. A combination of two or more various types of fibers in a single plastic gives a hybrid composite and it is mainly used in the aerospace industry. Sometimes, a ceramic layer or steel material may be bonded to the surface of other metallic structures and act as a thermal barrier in a high temperature environment. The sudden change in the material properties across the bonded region produces a stress jump and may further give rise to delamination or cracking of the interface. One way to overcome this shortcoming is to employ an FGB beam in which the material properties vary continuously and thereby possess noticeable advantages over homogeneous and layered materials in maintaining the integrity of the structure.

In order to design these types of structures, their dynamic analysis needs to be investigated. The dynamic analysis char- acteristics of rotating FML, HCB, and FGB beams differ from those of non-rotating structures. The centrifugal inertial force due to the rotational motion causes the increment of the bending stiffness of the structure, which naturally results in the variation of natural frequencies. Sinmazcelik et al. ${ }^{1}$ explained and reviewed the different types bonding and different testing methods on different FML. Huang et al., proposed a discrete method for the analysis of flap-wise bending vibration of rectangular plates using Dirac's delta function. ${ }^{2}$ The effects of the positions of point supports, the variable thickness, the aspect ratio, and the boundary conditions on the frequencies were studied. Hashemi et al. studied the effect of different parameters, including the aspect ratio, thickness ratio, hub radius ratio, and rotation speed on the natural frequencies of rotating thick plates by using the Mindlin-Reissner plate theory along with second order strain-displacement assumptions that were applied for plate modeling. ${ }^{3}$ The Kane dynamic method was employed for the derivation of nonlinear governing equations of motion, which included the Coriolis effects and the couplings between in-plane and out of plane deformations. The free vibration of rotating tapered cantilever Bernoulli-Euler beams with linearly varying rectangular cross-section was studied by Ozdemir and Kaya by using differential a transform method. ${ }^{4}$ For rotating Euler beams at high angular velocities, Huang et 
al. calculated the natural frequency of the free vibration and the coupled lag wise bending and axial vibration was investigated. ${ }^{5}$ The free vibration analysis of rotating tapered beams was investigated by Banerjee et al. by developing a dynamic stiffness matrix and a detailed set of numerical results were presented. ${ }^{6}$ In the experiments conducted by Alijani et al. on the non-linear response of sandwich plates made from carbon/epoxy laminate using a laser Doppler vibrometer to obtain modal parameters. ${ }^{7}$ Rath and Sahu presented an experimental and numerical investigation on the free vibration behavior of laminated composite plates subjected to varying temperatures and moisture, and observed that there was a reduction in the natural frequency with the increase in temperature and moisture concentration in laminated composites. ${ }^{8}$ The linear and nonlinear free vibrations of rotating composite Timoshenko beams was studied by Arvin and Bakhtiari-Nejad by basing the formulation on the nonlinear Von-Karman strain-displacement relationships. ${ }^{9}$ A linear and non-linear natural frequency of free flexural vibration of symmetric laminated cross-ply rectangular composite plates with fixed, simply supported boundary conditions was calculated by using first order deformation theory along with the Galerkin method and the method of multiple scales by Razavi and Shooshtari. ${ }^{10}$ Khalili et al. studied the effects of the stacking sequence, fiber orientation, axial load, internal pressure, and geometrical parameters on the transient response of FML shells and found that for a specific range of aspect ratios. ${ }^{11}$ The free vibration analysis of FGB with simply supported edges was performed by Aydogu and Taskin with the assumption that the Young's modulus of the assumed beams varied in the thickness direction according to the power law and exponential law. ${ }^{12}$ Sina et al. developed a new beam theory for the free vibration analysis of shear deformable FGB beam and the results showed that the new theory was a little different in natural frequency from the traditional first order shear deformation beams theory and the mode shapes of the two methods are coincidental. ${ }^{13}$ Atmane et al. investigated free vibration analysis of variable cross section of a functionally graded beams and concluded that the non-uniformity in the cross-section and the homogeneity in material properties influenced the natural frequencies. ${ }^{14}$ Fakhari and Ohadi studied the free vibration analysis of functionally graded thick and annular plates with linear and nonlinear thickness variation along the radial direction by using the polynomial-Ritz method. ${ }^{15}$ The material properties of the functionally graded plates were assumed to be graded in the thickness direction according to the power law distribution in terms of the volume fractions of the constituents. Ziane et al. investigated the free vibration analysis of an FGB box beam with different boundary conditions on the basis of first-order shear deformation theory. ${ }^{24}$ Meiche et al. studied buckling and vibration analysis of functionally graded materials beam using the new hyperbolic shear deformation theory. ${ }^{25}$ Hebali et al. studied the static and free vibration analysis of functionally graded plates using new quasi three-dimensional hyperbolic shear deformation theories. ${ }^{26}$ Benachour et al. studied free vibration analysis of plates made of functionally graded materials with an arbitrary gradient using refined plate theory. ${ }^{27}$ Meziane et al. presented an efficient and simple refined shear deformation theory for the vibration and buckling of exponentially graded material sandwich plate resting on elastic foundations under various boundary conditions. ${ }^{28}$ Zidi et al. studied the bending response

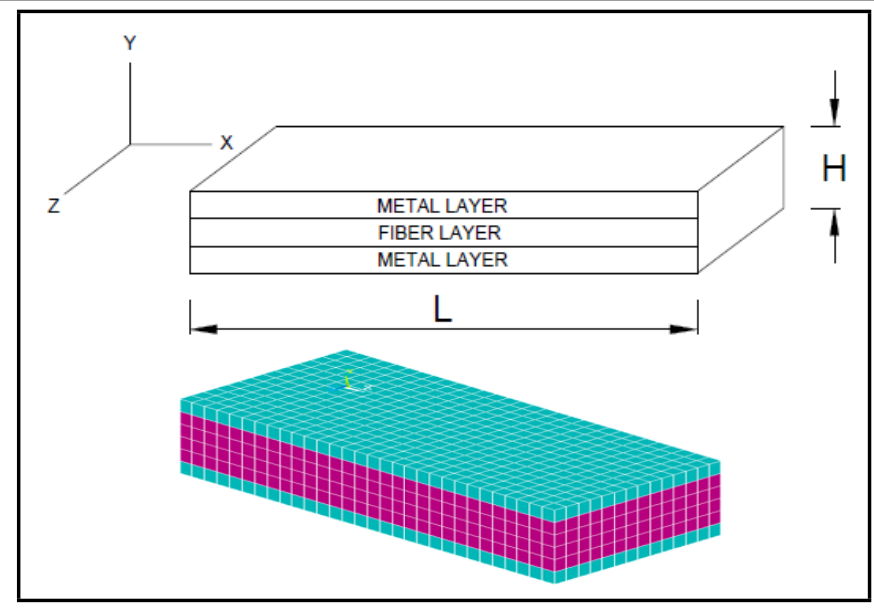

Figure 1. Schematic and finite element modeling of FML.

Table 1. Properties of isotropic materials.

\begin{tabular}{|c|c|c|c|}
\hline Materials & Aluminum & Magnesium & Titanium \\
\hline$E(\mathrm{GPa})$ & 70 & 45 & 116 \\
\hline$\nu$ & 0.28 & 0.29 & 0.32 \\
\hline$\rho\left(\mathrm{kg} / \mathrm{m}^{3}\right)$ & 2700 & 1738 & 4506 \\
\hline
\end{tabular}

of FGB beam resting on elastic foundation and subjected to hygro-thermo-mechanical loading. ${ }^{29}$ Larbi et al. developed a shear deformation beam theory based on neutral surface position for bending and free vibration analysis of functionally graded beams. ${ }^{30}$

The objective of this paper is to investigate the free vibration analysis of fiber metal laminates, hybrid composites, and functionally graded beams in rotating and non-rotating conditions by using finite element analysis. In this paper, we studied the effect of different metal alloys, namely aluminum, titanium, and magnesium, composite fibers (i.e. glass, carbon, and aramid), and different angular velocities, and the aspect ratio $(L / H)$ on free vibration analysis of rotating and non-rotating FML. The dynamic analysis of rotating and non-rotating hybrid composite beams was studied with different angular velocities and the aspect ratio $(L / H)$. The modal characteristic of rotating and non-rotating FGB beams was also studied with different homogenization methods for the computation of the material properties and different angular velocities. The current study is relevant to aero-structures.

\section{MATERIAL PROPERTIES AND FINITE EL- EMENT MODELING}

\subsection{Modeling of Fiber Metal Laminates}

FMLs consist of alternating layer of metals and composite fibers. The modeling was done by using commercial software ANSYS 14.5. The length, height, and width of the FML beams were $0.9 \mathrm{~m}, 0.3 \mathrm{~m}$, and $0.4 \mathrm{~m}$ respectively. The equal thickness of metals and fiber layers were taken for this study. The materials used for this modeling is shown in Tables1 and 2. The element chosen for this analysis was SHELL181 because it was suitable for analyzing thin to moderately-thick shell structures. It is a 4-node element with 6 degrees of freedom at each node, namely translations in the $x, y$, and $z$ directions and rotations about the $x, y$, and $z$ axes. The finite element modeling of fiber metal laminates is shown in Fig. 1. 


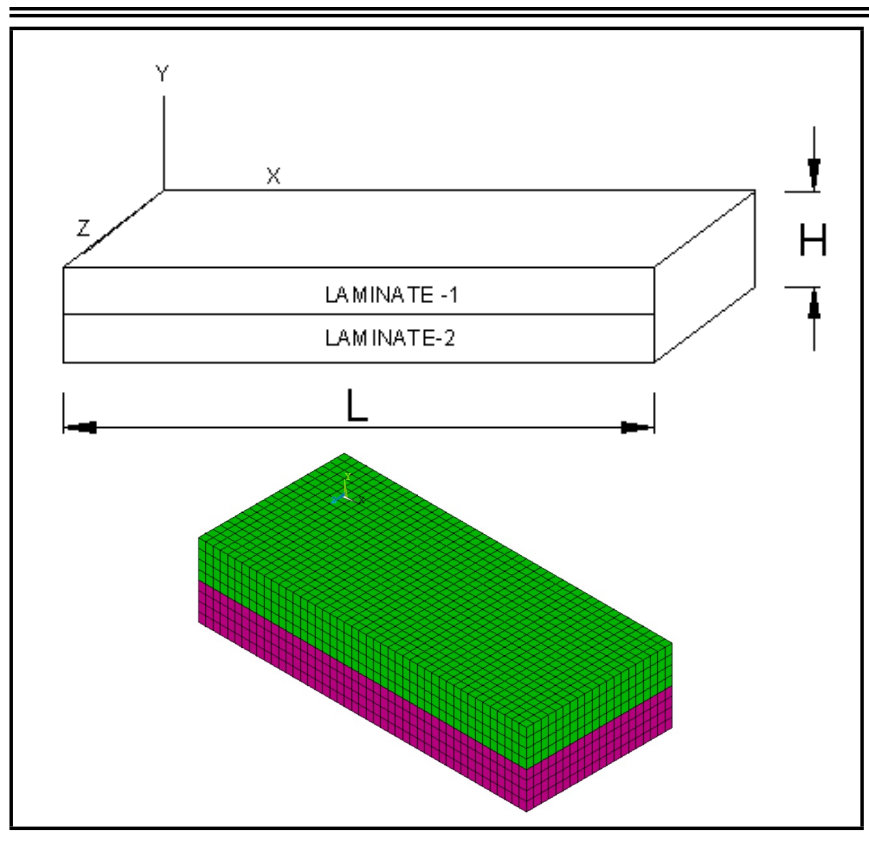

Figure 2. Schematic and finite element modeling of HCB.

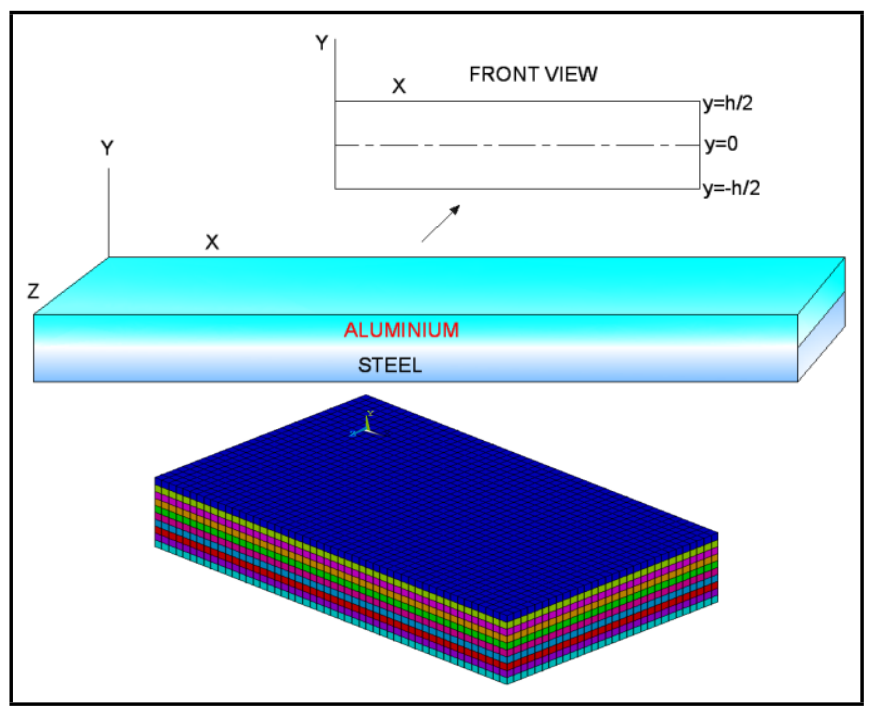

Figure 3. Schematic and finite element modeling of FGB beam.

\subsection{Modeling of Hybrid Composite Beam}

The hybrid composite beam consisted of two different composite fibers. The modeling of the HCB had been done using finite element analysis software ANSYS 14.5. The length and height of the hybrid composite beams were $0.9 \mathrm{~m}, 0.3 \mathrm{~m}$, and $0.4 \mathrm{~m}$ respectively. Fiber layers of equal thickness were considered for this study. The material used for this modeling and analysis is shown in Table 2. The element chosen for this dynamic analysis was SHELL 181 because it was suitable for analyzing thin to moderately-thick shell structures. It is a 4noded element with 6 degrees of freedom at each node namely translations in the $x, y$, and $z$ directions and rotations about the $x, y$, and $z$ axes. The finite element modeling of HCB is shown in Fig. 2.

\subsection{Modeling of Functionally Graded Beam}

The material properties of FGB beams were assumed to vary continuously through the thickness. The materials used for this beam were aluminum and steel. The bottom and top surfaces of the FGB beam were aluminum and steel-rich respectively. Three homogenization methods were deployable for the computation of material properties, such as Young's modulus $(E)$, Poisson's ratio $(v)$, and density $(\rho)$ namely: (1) the power law distribution, (2) the exponential distribution, and (3) the MoriTanaka scheme.

\subsubsection{Exponential Law constitutive Equations}

According to exponential law distribution, the material properties of FGB beams are characterized by Belabed et. al.: ${ }^{18}$

$$
\begin{aligned}
& E(z)=E_{t} e^{(-\delta(1-2 Z / h))} \\
& v(z)=v_{t} e^{(-\delta(1-2 Z / h))} \\
& \rho(z)=\rho_{t} e^{(-\delta(1-2 Z / h))}
\end{aligned}
$$

\subsubsection{Mori-Tanaka Scheme constitutive Equations}

In this study, the FGB beam made of aluminum, steel, and the effective material properties of the FGB beam (i.e., Young's modulus, Poisson's ratio, and mass density), varied continuously in the thickness direction ( $z$ axis direction). According to the Mori-Tanaka homogenization scheme, the material properties were calculated based on Benveniste ${ }^{19}$, Mori and Tanaka: ${ }^{20}$

$$
\begin{gathered}
E(z)=E_{S}+\left(E_{A}-E_{S}\right) \\
\cdot\left(\frac{V_{A}}{1+\left(1-V_{A}\right)\left(E_{A} / E_{S}-1\right)(1+v) /(3-3 v)}\right) \\
\rho(z)=\rho_{S}+\left(\rho_{A}-E \rho_{S}\right) \\
\cdot\left(\frac{V_{A}}{1+\left(1-V_{A}\right)\left(E_{\rho} / E_{\rho}-1\right)(1+v) /(3-3 v)}\right) .
\end{gathered}
$$

Where $V_{A}=(0.5+z / h)^{K}$ is the volume fraction of aluminum. According to Yang et al., the effect of variation Poisson's ratio $(\vartheta)$ on the response of FGB beams was very small: this material property assumed to be constant for convenience. ${ }^{21}$ Where $K$ was the non-negative variable parameter which dictated the material variation profile through the thickness of the beam $(K=1)$.

\subsubsection{Power Law Constitutive Equations}

The effective material properties of the FGB beams were assumed to be varying continuously along their thickness directions and were obtained by using the power law. The power law distribution of a panel considered from the midplane reference plane could be written according to Belabed et al. (2014):

$$
V_{f}=\left(\frac{y}{h}+\frac{1}{2}\right)^{n}
$$

Where $n$ is the power-law gradient, $0 \leq n \leq \infty$. The functionally graded material with two constituents and their properties, such as Young's modulus $(E)$, Poisson's ratio $(v)$ and the mass density $(\rho)$ were obtained using the following equations:

$$
E=\left(E_{a}-E_{s}\right)\left(\frac{y}{h}+\frac{1}{2}\right)^{n}+E_{s}
$$


Table 2. Properties of orthotropic materials. Lei et al., ${ }^{17}$ Malik and Arif. ${ }^{16}$

\begin{tabular}{|c|c|c|c|c|c|c|c|c|c|c||}
\hline Materials & $\begin{array}{c}E_{x} \\
(\mathrm{GPa})\end{array}$ & $\begin{array}{c}E_{y} \\
(\mathrm{GPa})\end{array}$ & $\begin{array}{c}E_{z} \\
(\mathrm{GPa})\end{array}$ & $v_{x y}$ & $v_{y z}$ & $v_{x z}$ & $\begin{array}{c}G_{x y} \\
(\mathrm{GPa})\end{array}$ & $\begin{array}{c}G_{y z} \\
(\mathrm{GPa})\end{array}$ & $\begin{array}{c}G_{x z} \\
(\mathrm{GPa})\end{array}$ & $\begin{array}{c}\rho \\
\left(\mathrm{kg} / \mathrm{m}^{3}\right)\end{array}$ \\
\hline Carbon/Epoxy & 60.8 & 58.2 & 58.2 & 0.07 & 0.4 & 0.07 & 4.5 & 5 & 4.55 & 1600 \\
\hline Glass/Epoxy & 26 & 26 & 8 & 0.1 & 0.25 & 0.25 & 3.8 & 2.8 & 2.8 & 1800 \\
\hline Aramid/Epoxy & 67 & 4.7 & 4.7 & 0.34 & 0.34 & 0.45 & 2 & 1.58 & 2 & 1440 \\
\hline
\end{tabular}

Table 3. Convergence study on finite elements for FML, HCB and FGB beams.

\begin{tabular}{||c|c|c|c|}
\hline Total number & \multicolumn{3}{|c|}{ Fundamental Natural Frequency (Hz) } \\
\cline { 2 - 4 } of elements & FML & HCB & FGB \\
\hline 100 & 226.48 & 215.84 & 386.61 \\
\cline { 1 - 1 } & 226.28 & 215.52 & 386.11 \\
\cline { 1 - 1 } 200 & 226.24 & 215.50 & 386.00 \\
\cline { 1 - 1 } 200 & 226.23 & 215.48 & 385.97 \\
\cline { 1 - 1 } 1600 & 226.22 & 215.47 & 385.95 \\
\cline { 1 - 1 } 2500 & 226.22 & 215.47 & 385.95 \\
\hline 3600 &
\end{tabular}

$$
\begin{aligned}
& \rho=\left(\rho_{a}-\rho_{s}\right)\left(\frac{y}{h}+\frac{1}{2}\right)^{n}+\rho_{s} ; \\
& v=\left(v_{a}-v_{s}\right)\left(\frac{y}{h}+\frac{1}{2}\right)^{n}+v_{s} .
\end{aligned}
$$

The power law distribution was used for the continuous gradation of material properties in the thickness direction. The effective material properties were calculated based on Eqs. (7), (8), and (9), when $y=-h / 2, E=E_{s}, \rho=\rho_{s}$ and similarly, when $y=+h / 2, E=E_{a}, \rho=\rho_{a}$ (i.e. the material properties varied continuously from steel at the bottom surface to aluminum at the top surface). The element chosen for this analysis was also SHELL281, which is a layered version of the 8-node structural shell model. The finite element modeling of FGB and schematic diagram is shown in the Fig. 3.

\section{NUMERICAL RESULTS AND DISCUSSIONS}

A numerical analysis was carried out to analyze the free vibration analysis of fiber metal laminates, hybrid composite, and functionally graded beams. Moderately thin beams were considered for the study, namely the length, height, and width of the beams, which were $0.9 \mathrm{~m}, 0.3 \mathrm{~m}$, and $0.4 \mathrm{~m}$ respectively. The beam was divided into a number of layers in the thickness direction and each layer was assumed to be isotropic. The influence of the aspect ratio, different fibers and metal alloys were studied against the natural frequency for both rotating and non-rotating in fiber metal laminates. In hybrid composite beams the effect of aspect ratio and different fibers are studied against natural frequency studied for both rotating and nonrotating beams. The effect of angular velocity was studied in a functionally graded materials beam against the natural frequency in different material distribution methods. Boundary conditions for the beams were fixed-free.

\subsection{Convergence study}

Table 3 shows a convergence study of fiber metal laminates, hybrid composites, and functionally graded beams with clamped free boundary conditions, $L / h=3$, and power law material properties (power law gradient $n=1$ ) were taken for functionally graded beams. All the results given here after corresponded to beams with 2500 finite elements.

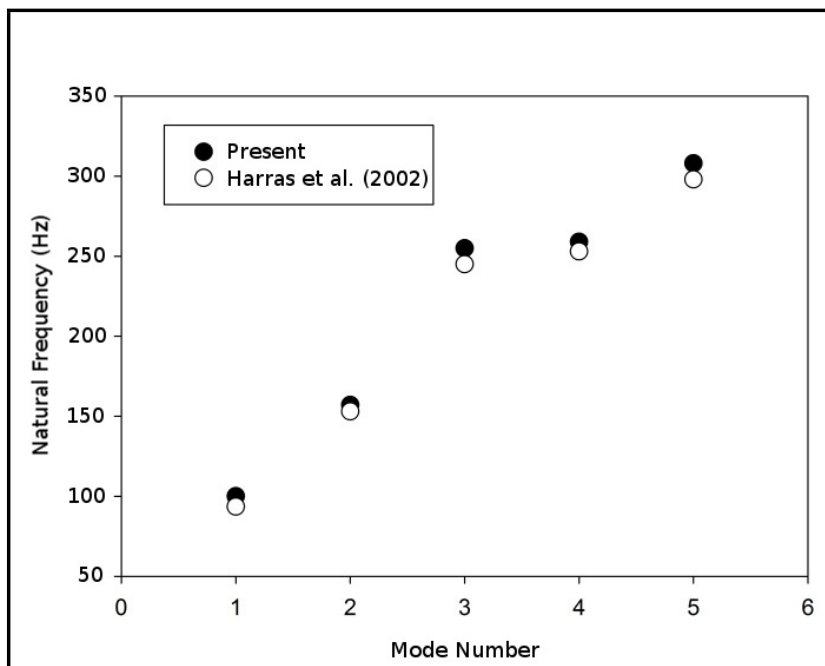

a) Fiber Metal Laminates

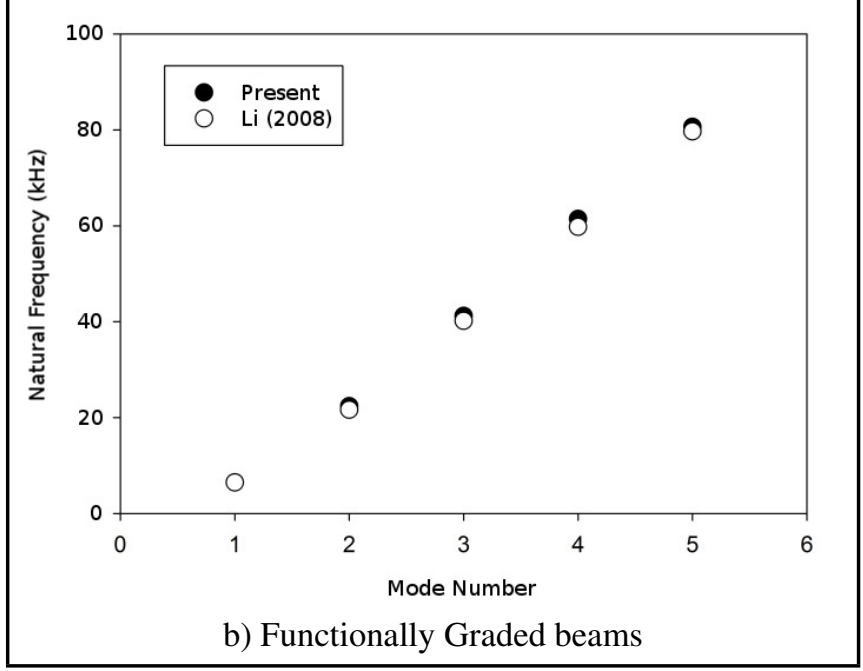

Figure 4. Finite element analysis validations with experimental results.

\subsection{Comparison with Experimental Studies}

This section compares the results of this study with the results available in literatures. A modal analysis of a non- rotating fixed plate of GLARE 3 hybrid laminate lay-up with a cross ply $(0,90)$ orientation of three metal layers of thickness $0.3 \mathrm{~mm}$ and alternate fibers layers of thickness $0.25 \mathrm{~mm}$ was performed and the results were compared with the experimental values available in the literature Harras et al. ${ }^{22}$ Similarly the first five natural frequencies of a simply supported FGB (length $L=0.5 \mathrm{~m}$ and depth $h=0.125 \mathrm{~m}$ ) consisting of a steel rich bottom layer and an aluminum rich top layer, with power law gradient $(n)$ of 1 was obtained using modal analysis. The obtained numerical results were compared with the results obtained by $\mathrm{Li}^{23}$ Fig. 4, shows a plot of natural frequency against mode number comparing it. 


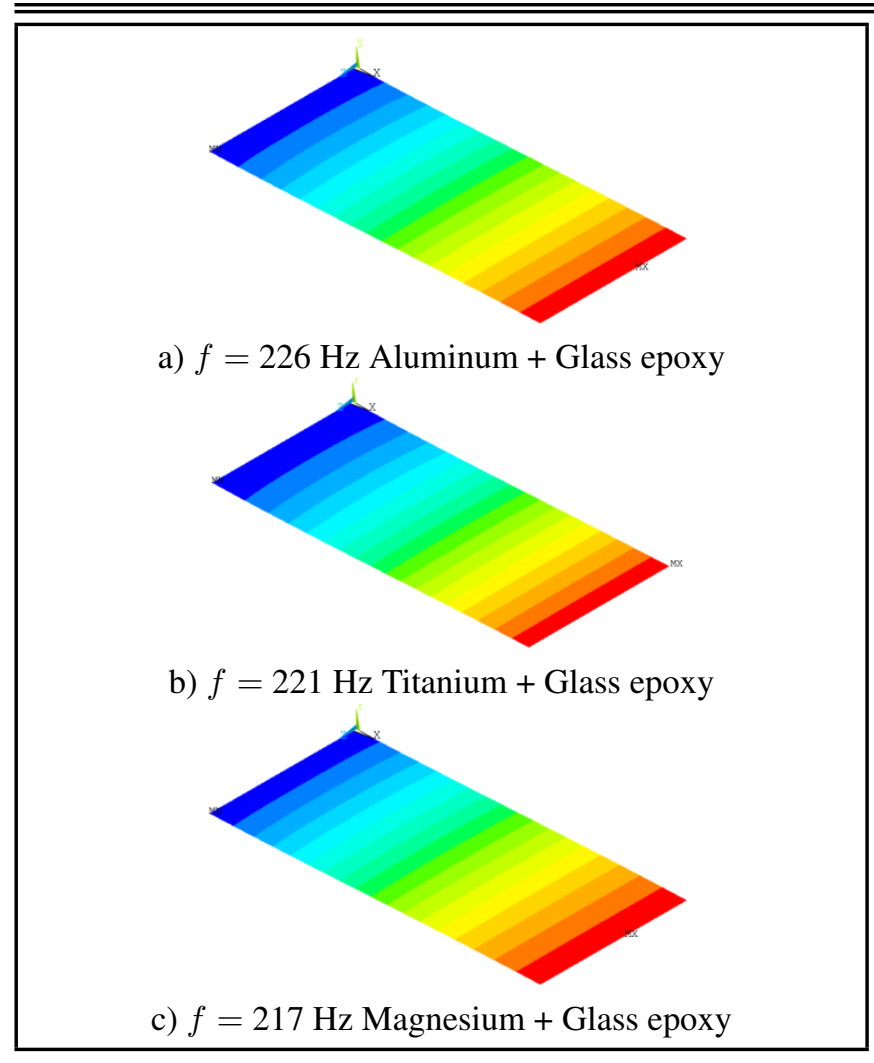

Figure 5. Variation of fundamental natural frequency of glass epoxy with different metal alloys.

\subsection{Flapwise bending vibration analysis of non-rotating FML}

Figure 5 shows the first natural frequency of FML with an aspect ratio $(L / H)$ of 3 and an FML of 2/4 (two metal layers + four fibers) that had an orientation angle of 0/30/45/90 with fixed-free boundary conditions. It was observed that there was no significant difference in the mode shape for the different metal alloys, but the natural frequency was highest for GLARE (glass-reinforced aluminum laminate), followed by that with titanium and finally magnesium based FML. Figure 6 shows the variation of fundamental natural frequency of $\mathrm{Al}, \mathrm{Ti}$ and Mn based FML against aspect ratios.

\subsubsection{Flapwise bending vibration analysis of rotating FML}

Figure 7 depicts the first natural frequency of FML 2/4, with an aspect ratio $(L / H)$ of 3 , having an orientation angle of $0 / 30 / 45 / 90$ with an angular velocity of $500 \mathrm{rad} / \mathrm{sec}$. It was observed that there was no significant difference in the mode shape for the different metals. The natural frequency was higher for GLARE when compare to other two kind of FMLs. On comparing the natural frequency of the non-rotating counterparts, it was observed that the frequencies had increased. This could be attributed to the increase in the stiffness of the beams (motion along flap wise direction was reduced) due to the effect of centrifugal force upon rotation. Figures 8, 9, and 10 , show that the fundamental natural frequency of rotating FML 2/4 against an aspect ratio with different metal alloys and an orientation angle of $0 / 30 / 45 / 90$. The fundamental natural frequency decreased when there was an increase in the aspect ratio due to the softening effect resulting from the decrease in cross sectional area and also as the rotational speed increased,

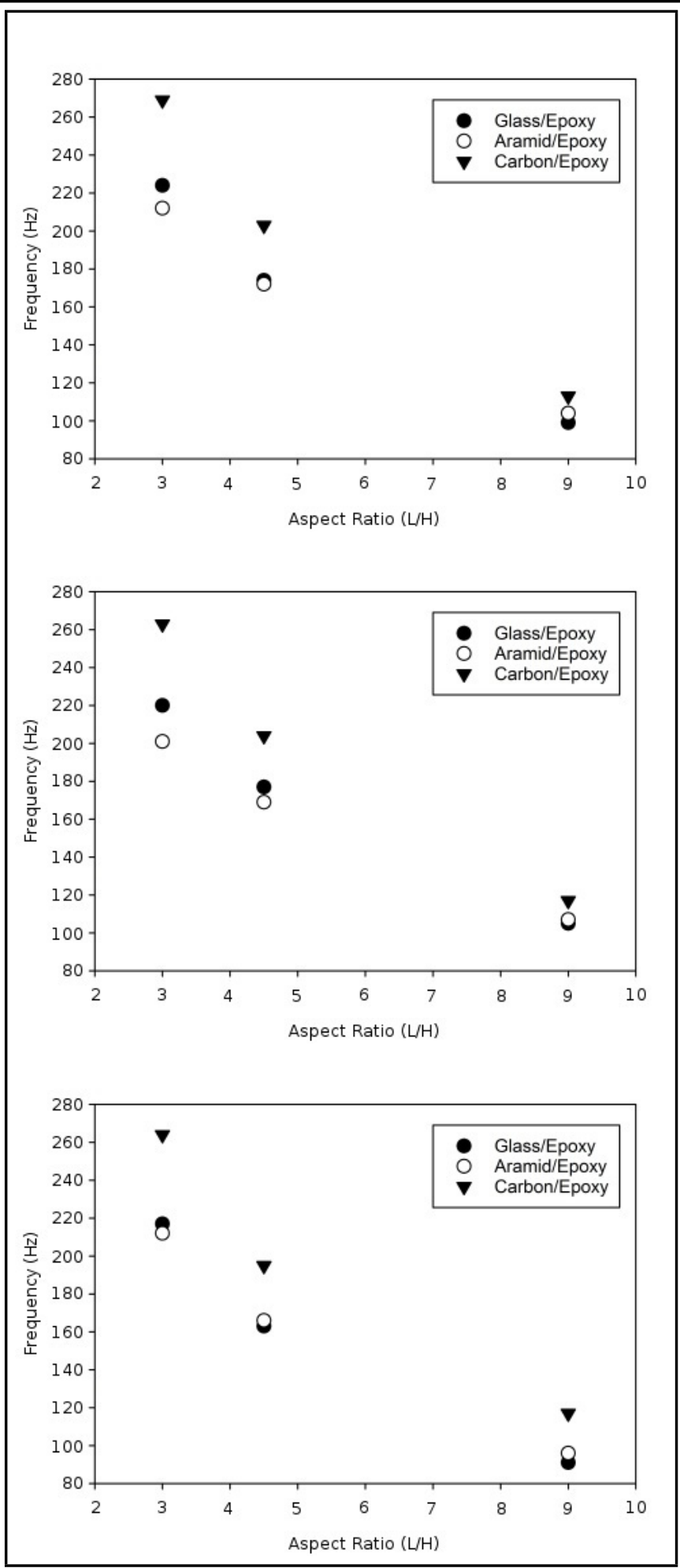

Figure 6. Variation of fundamental natural frequency of $\mathrm{Al}, \mathrm{Ti}$ and $\mathrm{Mn}$ based FML against aspect ratios.

the centrifugal force increased and as a result, the natural frequency also increased. as such, GLARE had the highest fundamental frequency among other kind of FMLs.

\subsection{Free vibration analysis of non-rotating and rotating HCB}

Figures 11, 12, and 13, show the fundamental natural frequency of a $\mathrm{HCB}$ with an aspect ratio $(L / H)$ of 3 having an orientation angle of 0/30/45/90 for conditions of non-rotating, 


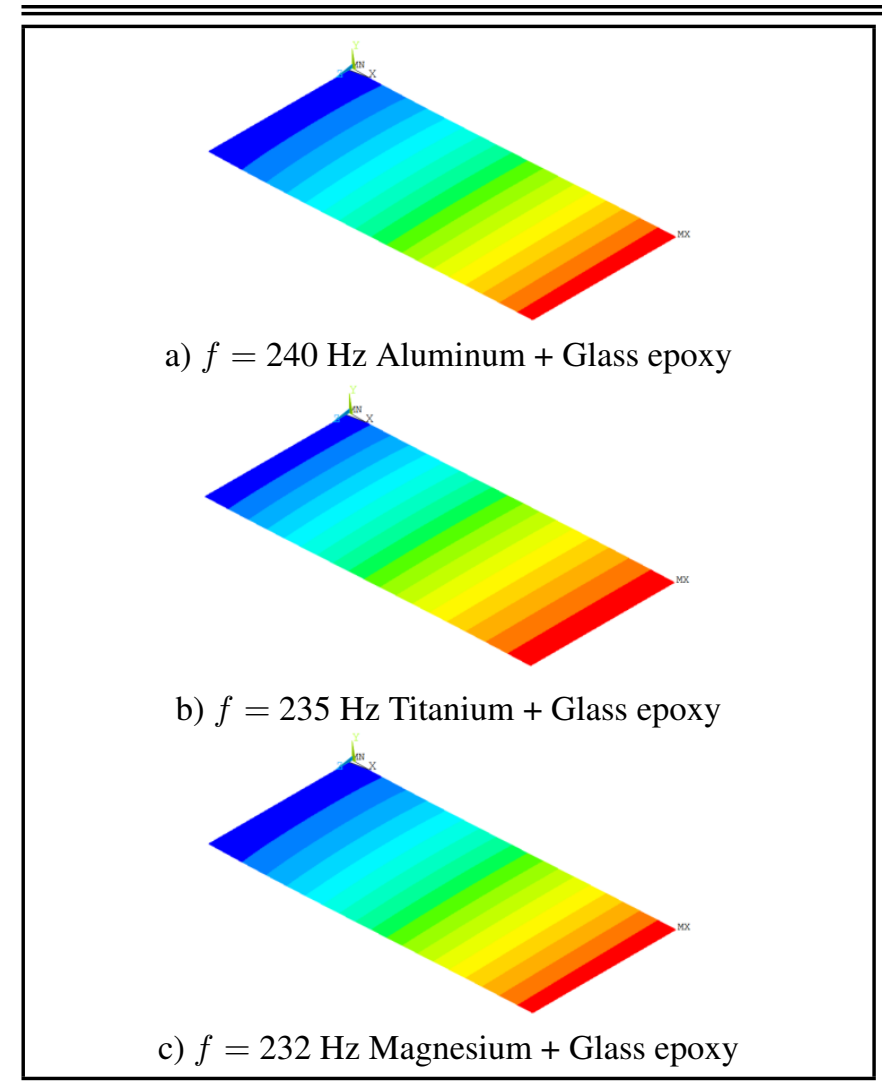

Figure 7. Variation of fundamental natural frequency of glass epoxy based FML with angular velocity of $500 \mathrm{rad} / \mathrm{sec}$.

rotating at $500 \mathrm{rad} / \mathrm{s}$, and rotating at $1000 \mathrm{rad} / \mathrm{sec}$ respectively. It was observed that there was nothing significant in the mode shape for the different hybrid composite beam. The natural frequency was higher for the glass-carbon hybrid beam when compare to other two kind of HCBs. Because glass-carbon hybrid beams have a lot of strength compared to carbon-aramid and glass-aramid hybrid beams. On comparing the natural frequency of non-rotating hybrid beams, the frequencies of rotating hybrid beams had a high frequency. This could be attributed to the increase in the stiffness of the beams due to the effect of centrifugal force upon rotations. Figure 14, shows that the fundamental natural frequency of rotating HCB against different aspect ratios, angular velocity, and orientation angle $0 / 30 / 45 / 90$. The fundamental natural frequency decreases when increases in the aspect ratio and also the rotational speed increases the centrifugal force increases and as a result the natural frequency also increases.

\subsection{Free vibration analysis of non-rotating and rotating FGM}

\subsubsection{Power law distribution}

Figures 15 and 16, show that the first three mode shapes of non-rotating FGM beam for fixed-free with power law gradient as 1 and rotating FGB beam with an angular velocity of 500 $\mathrm{rad} / \mathrm{sec}$ with power law gradient as 1 respectively. As discussed before, the centrifugal force affected the natural frequencies and hence frequencies of rotating beams are higher than that of non-rotating beams.

Figure 17 shows the first five natural frequencies of nonrotating and rotating FGB: it was observed that the frequencies of linearly interpolated properties $(n=1)$ was higher than
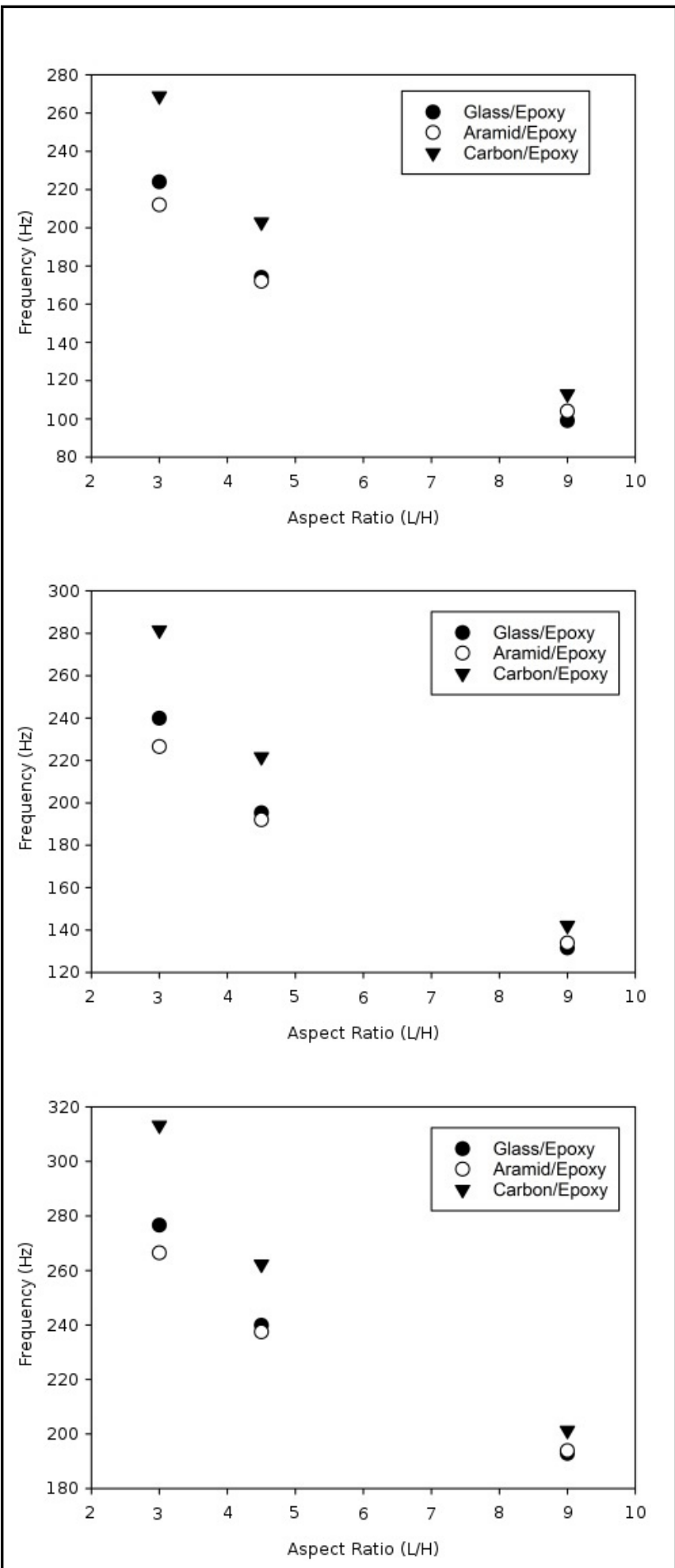

Figure 8. Variation of fundamental natural frequency of aluminum based FML-2/4 with different angular velocity.0, 500, and $1000 \mathrm{rad} / \mathrm{sec}$.

the quadratically interpolated properties $(n=2)$. The average Young's modulus for linear interpolation was $140 \mathrm{GPa}$ and 120.9 GPa for the quadratic interpolation. This reinforced the inference for laminates with higher stiffness, the natural frequencies increased. It was also observed that the rotating FGB beam had a higher natural frequency than the non-rotating one due to centrifugal force action along the longitudinal direction. For the higher rotational speed, there was not much change in the fundamental natural frequency. 


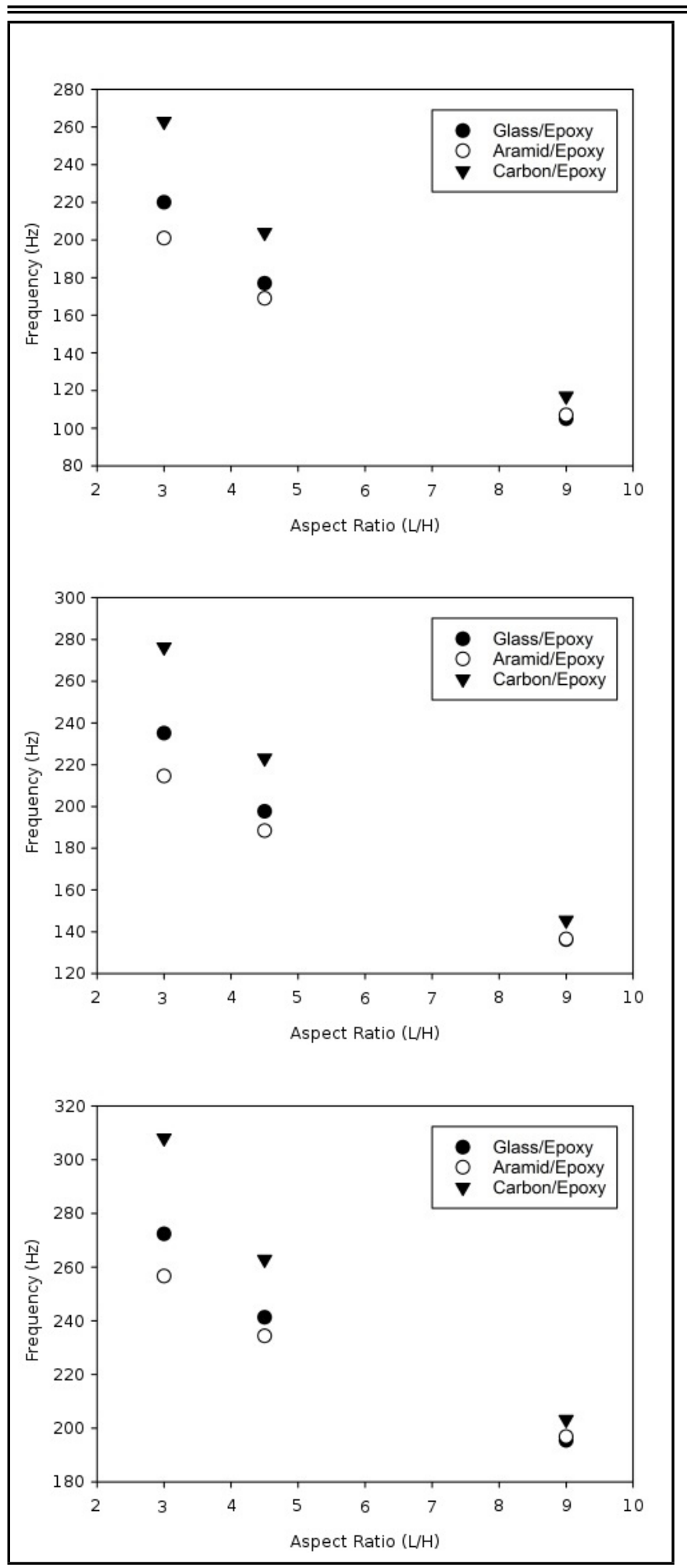

Figure 9. Variation of fundamental natural frequency of titanium based FML$2 / 4$ with different angular velocity. 0,500 , and $1000 \mathrm{rad} / \mathrm{sec}$.

\subsubsection{Exponential law distribution and Mori Tanaka's Scheme}

Figure 18 shows the comparison of first five natural frequencies of non-rotating and rotating FGB beams using exponential law and Mori Tanaka's Scheme with a fixed-free boundary condition. As discussed before, the centrifugal force affected the natural frequencies. Hence, the frequencies of rotating beams were higher than that of non-rotating beams in functionally graded beams in each Exponential and Mori Tanaka's

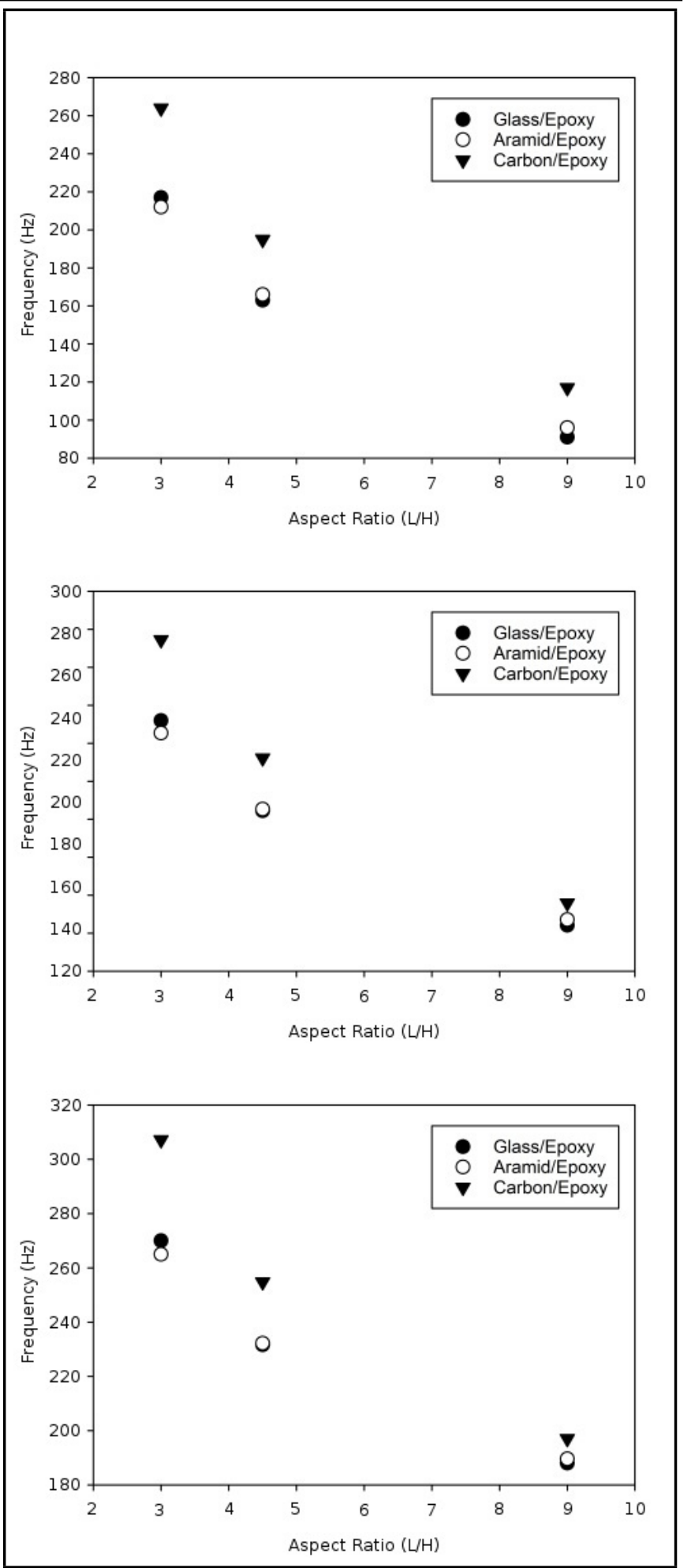

Figure 10. Variation of fundamental natural frequency of magnesium based FML-2/4 with different angular velocity 0,500 , and $1000 \mathrm{rad} / \mathrm{sec}$.

scheme. From the Fig. 18, it was observed that there were no changes in the fundamental natural frequency in different homogenization methods for the computation of the material properties.

\section{CONCLUSIONS}

The free vibration analysis of both rotating and non-rotating of fiber metal laminates, hybrid composite, and functionally graded material beams was studied. GLARE shows the highest 


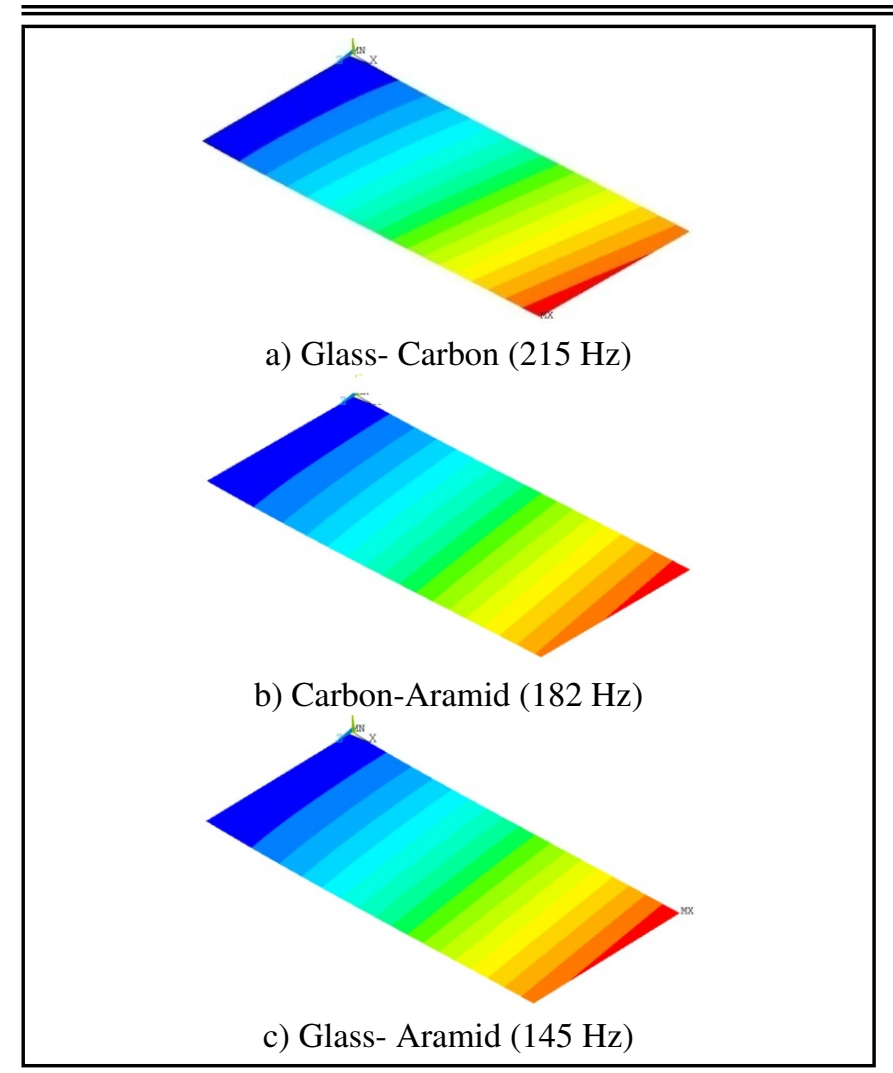

Figure 11. Variation of fundamental natural frequency of HCB with angular velocity $0 \mathrm{rad} / \mathrm{sec}$.

frequency among other kind of glass/epoxy based fiber metal laminates during free vibration analysis of non-rotating conditions. Carbon/epoxy based fiber metal laminates have a higher frequency compared to any other fiber based fiber metal laminates for any aspect ratio. It is observed also that the fundamental frequency increases as the aspect ratio decreases in fiber metal laminates. It has also been observed that glasscarbon hybrid beams have a higher frequency compared to other kinds of hybrid composite beams. It has been observed that the natural frequency decreases as the power law gradient increases in functionally graded beams. It has also been observed that the natural frequency increases when the angular velocity increases.

\section{REFERENCES}

1 Sinmazclik, T., Avcu, E., Bora, M. O., and Coban, O. A review: Fibers metal laminates, background, bonding types and applied test methods, Materials \& Design, 32 (36), 7185, (2011). http://dx.doi.org/10.1016/j.matdes.2011.03.011

2 Huanga, M., Mab, X. O., Sakiyamaa, T., Matsudaa, H., and Morita, C. Free vibration analysis of rectangular plates with variable thickness and point supports, Journal of Sound and Vibration, 300 (3-5), 435-452, (2007). http://dx.doi.org/10.1016/j.jsv.2005.01.059

3 Hashemi, S. H., Farhadi, S., and Carra, S. Free vibration analysis of rotating thick plates, Journal of Sound and Vibration, 323 (1-2), 366-384, (2009). http://dx.doi.org/10.1016/j.jsv.2008.12.007

4 Ozdemir, O. and Kaya, M, O. Flapwise bending vibration analysis of a rotating tapered cantilever Bernoulli-

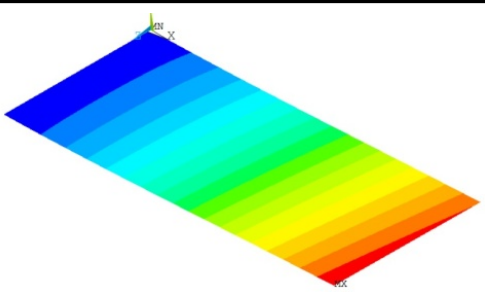

a) Glass- Carbon $(230 \mathrm{~Hz})$

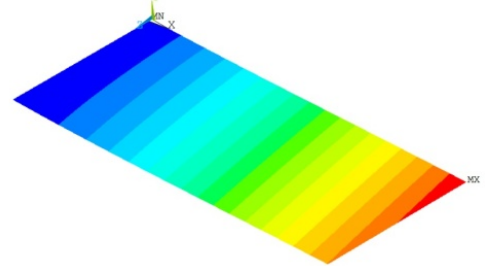

b) Carbon-Aramid(200 Hz)

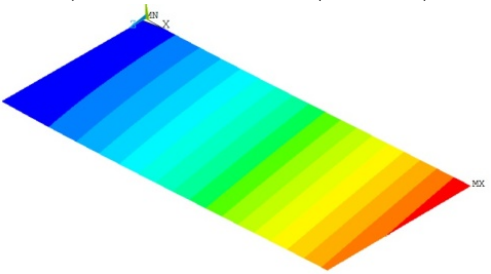

c) Glass- Aramid( $165 \mathrm{~Hz})$

Figure 12. Variation of fundamental natural frequency $\mathrm{HCB}$ with angular velocity $500 \mathrm{rad} / \mathrm{sec}$.

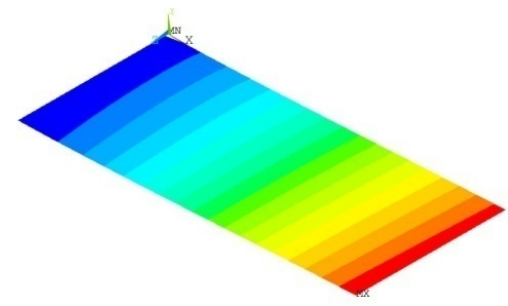

a) Glass- Carbon $(270 \mathrm{~Hz})$

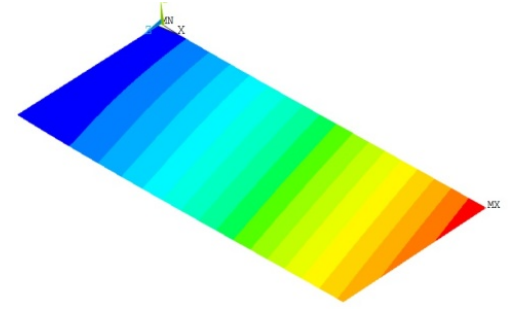

b) Glass- Carbon $(240 \mathrm{~Hz})$

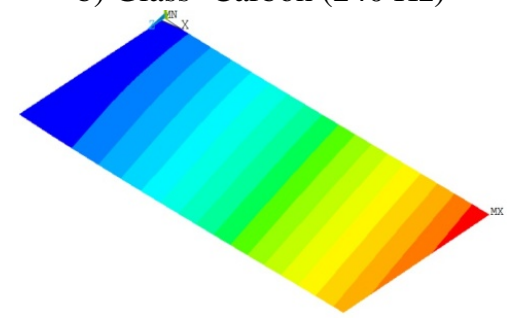

c) Glass- Carbon $(212 \mathrm{~Hz})$

Figure 13. Variation of fundamental natural frequency HCB with angular velocity $1000 \mathrm{rad} / \mathrm{sec}$.

Euler beams by differential transform method, Journal of Sound and Vibration, 289 (1-2), 413-420, (2006). http://dx.doi.org/10.1016/j.jsv.2005.01.055

5 Huang, C. L., Lin, W. Y., and Hsiao, K. M. Free vibra- 


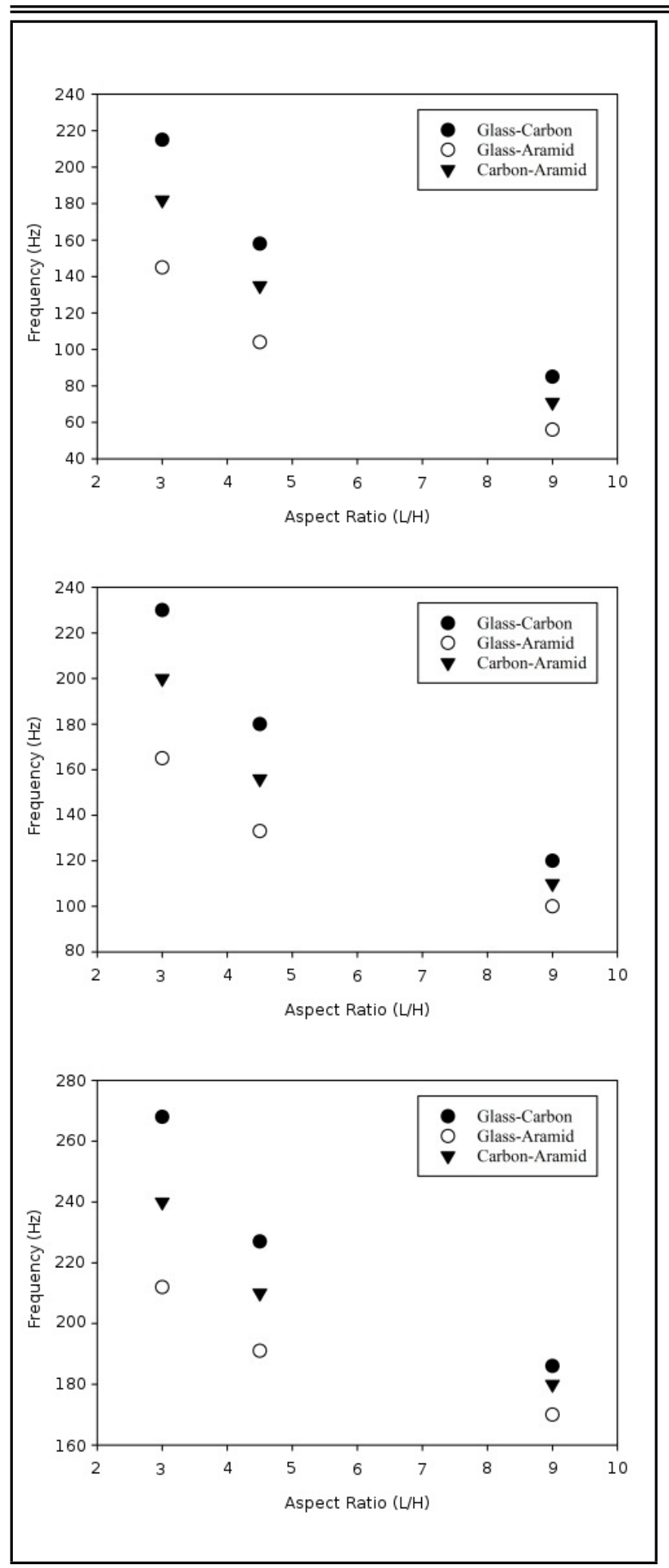

Figure 14. Variations of fundamental natural frequency of HCB with different angular velocity 0,500 , and $1000 \mathrm{rad} / \mathrm{sec}$.

tion analysis of rotating Euler beams at high angular velocity, Composite Structures, 88 (17-18), 991-1001, (2010). http://dx.doi.org/10.1016/j.compstruc.2010.06.001

6 Banerjee, J. R., Su, H., and Jackson, D. R. Free vibration of rotating tapered beams using the dynamic stiffness method, Journal of Sound and Vibration, 298 (4-5),10341054, (2006). http://dx.doi.org/10.1016/j.jsv.2006.06.040

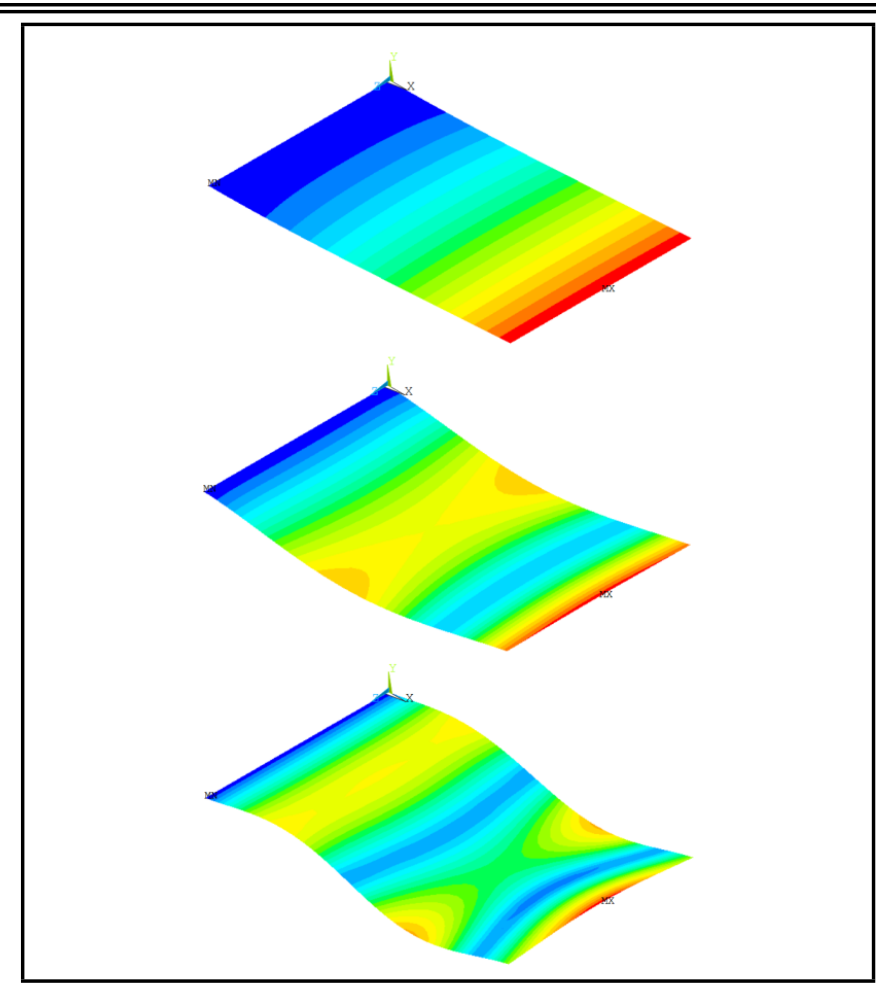

Figure 15. Variation of first three natural frequencies of non-rotating FGB beam.

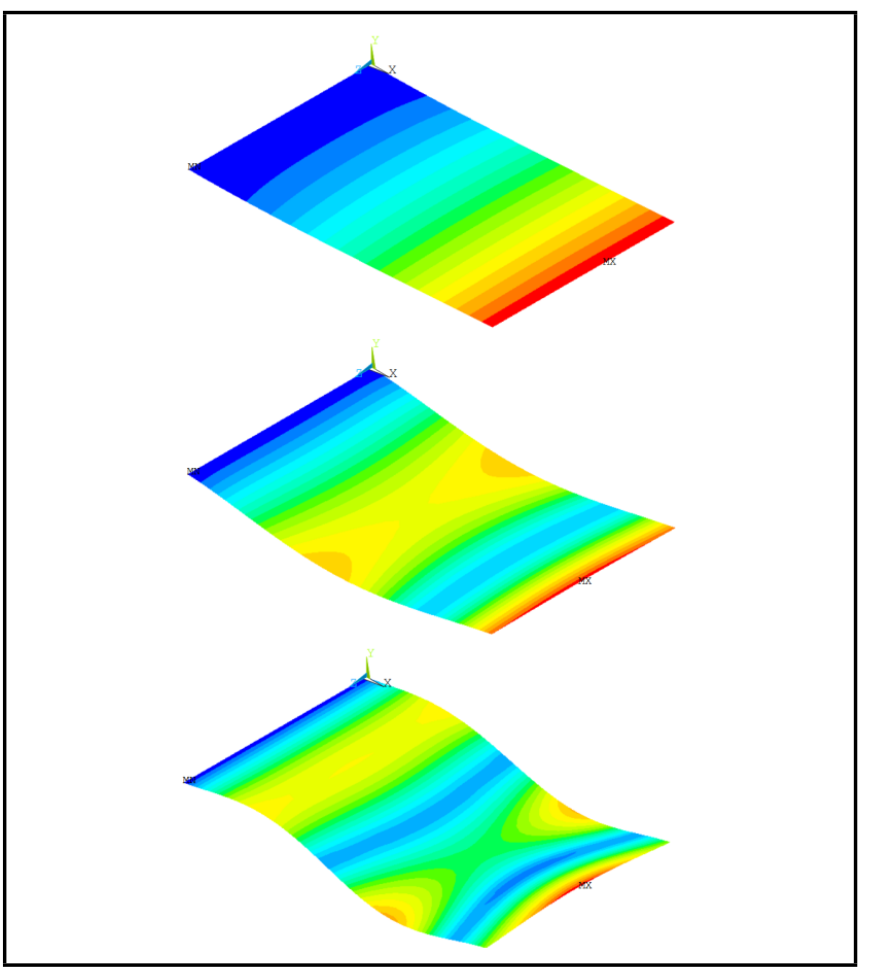

Figure 16. Variation of first three natural frequency of rotating FGB beam.

7 Alijani, F., Amabili, M., Ferrari, G., and D’Alessandro, V. Nonlinear vibrations of laminated and sandwich rectangular plates with free edges. Part 2: Experiments \& comparisons, Composite Structures, 105 (4), 37-45, (2013). http://dx.doi.org/10.1016/j.compstruct.2013.05.020

8 Rath, M. K. and Sahu, S. K. Vibration of woven fiber laminated composite plates in hydrothermal environment, Journal of Vibration and Control, 18 (13), 1957-1970, (2011). http://dx.doi.org/10.1177/1077546311428638 


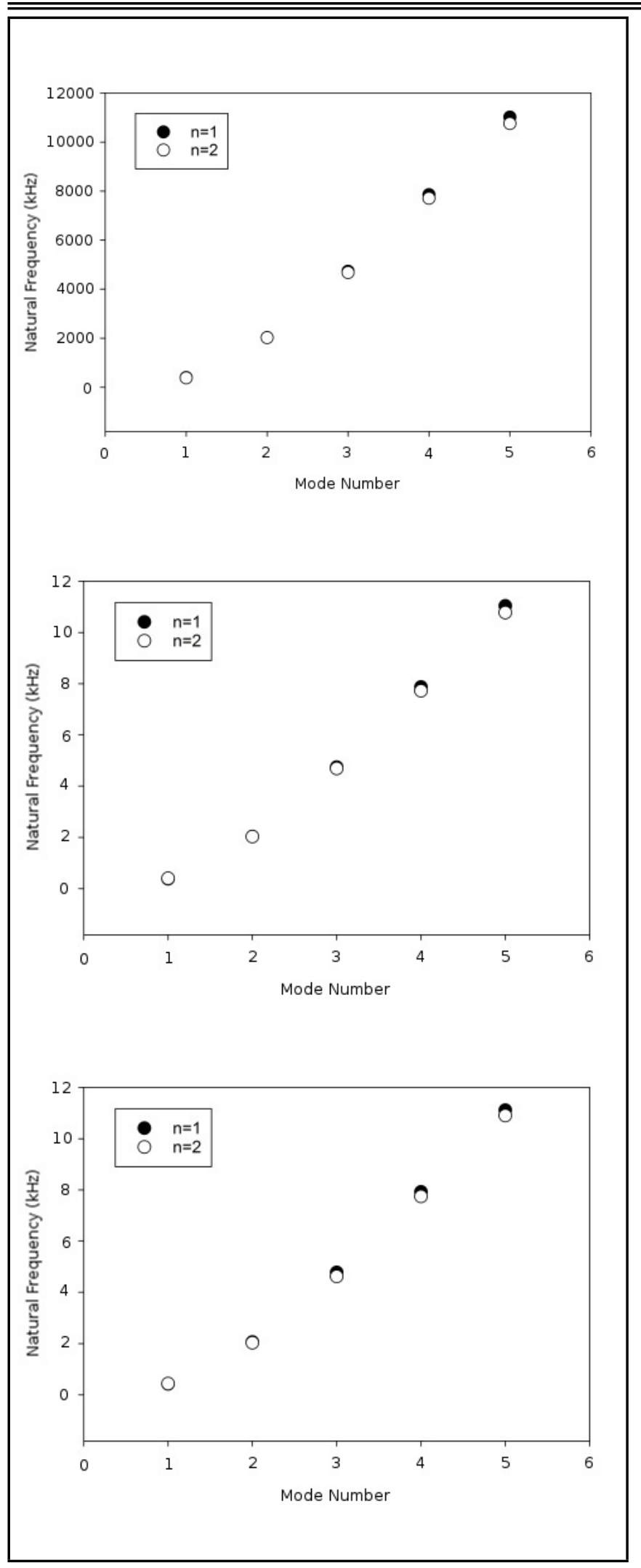

Figure 17. Variation of first five natural frequencies of rotating FGB beam $(n=1)$ against different angular velocity 0,500 , and $1000 \mathrm{rad} / \mathrm{sec}$.

9 Arvin, H. and Bakhtiari-Nejad, F. Nonlinear free vibration analysis of rotating composite Timoshenko beams, Composite Structures, 96, 29-43, (2013). http://dx.doi.org/10.1016/j.compstruct.2012.09.009

10 Razavi, S. and Shooshtari, A. A closed form solution for linear and nonlinear free vibrations of

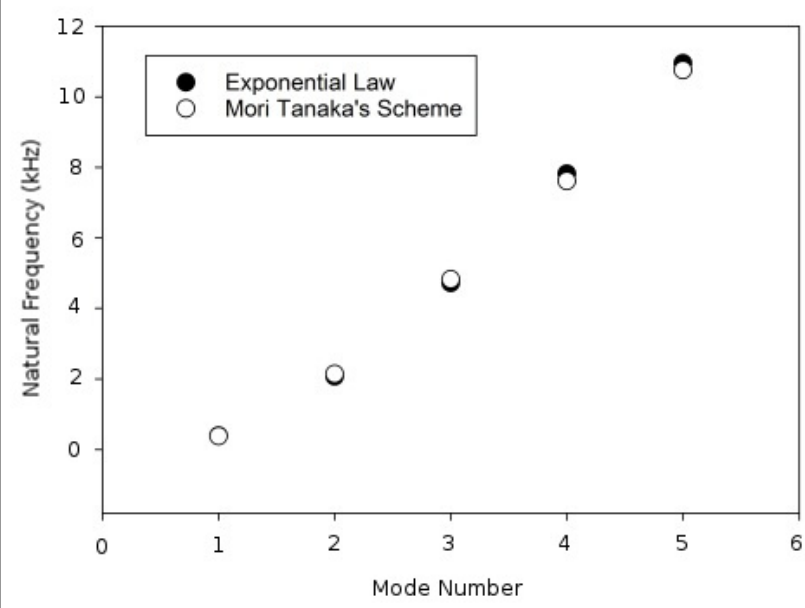

a) $0 \mathrm{rad} / \mathrm{sec}$

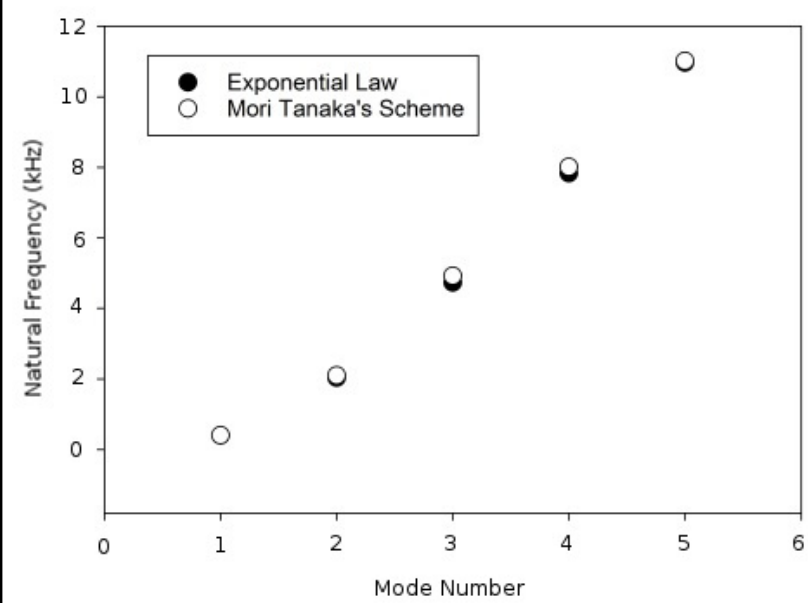

b) $500 \mathrm{rad} / \mathrm{sec}$

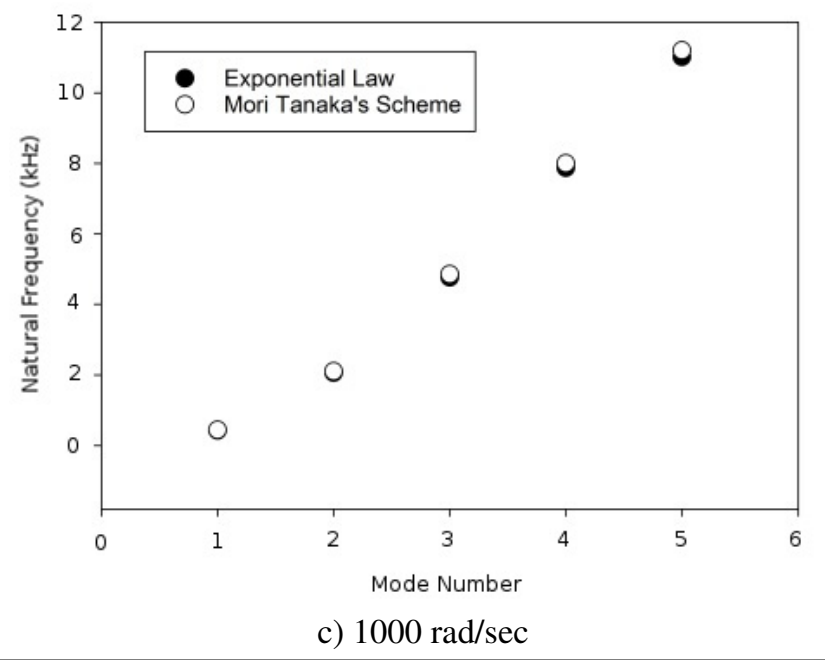

Figure 18. Variation of first five natural frequencies of rotating FGB beam using exponential and Mori- Tanaka's Scheme against different angular velocity.

composite and fiber metal laminated rectangular plates, Composite Structures, 92 (26), 63-75, (2010). http://dx.doi.org/10.1016/j.compstruct.2010.04.001

11 Khalili, S. M. R., Malekzadeh, K., Davar, A., and Mahajan, P. Dynamic response of pre-stressed fibers metal lami- 
nate circular cylindrical shells subjected to lateral pressure pulse loads, Composite Structures, 92 (130), 8-17, (2010). http://dx.doi.org/10.1016/j.compstruct.2009.11.012

12 Aydogdu, M. and Taskin, V. Free vibration analysis of functionally graded beams with simply supported edges, Materials \& Design, 28 (5), 1651-1656, (2007). http://dx.doi.org/10.1016/j.matdes.2006.02.007

13 Sina, S. A., Navazi, H. M., and Haddadpour, H. An analytical method for free vibration analysis of functionally graded beams, Materials \& Design, 30 (3), 741-747, (2009). http://dx.doi.org/10.1016/j.matdes.2008.05.015

14 Atmane, H. A., Tounsi, A., Meftah, S. A., and Belhadj, H. A. Free vibration behavior of exponential functionally graded beams with varying cross-section, Journal of Vibration and Control, 17 (2), 311-318, (2011). http://dx.doi.org/10.1177/1077546310370691

15 Fakhari, V. and Ohadi, A. Three-dimensional vibration analysis of functionally graded thick, annular plates with variable thickness via polynomial-Ritz method, Journal of Vibration and Control, 18 (11), 1698-1707, (2011). http://dx.doi.org/10.1177/1077546311403789

16 Malik, M. H. and Arif, A. F. M. ANN prediction model for composite plates against low velocity impact loads using finite element analysis, Composite Structures, 101, 290-300, (2013). http://dx.doi.org/10.1016/j.compstruct.2013.02.020

17 Yanga, L., Yana, Y., and Kuangb, N. Experimental and numerical investigation of Aramid fibers reinforced laminates subjected to low velocity impact, Polymer Testing, 32 (7), 1163-1173, (2013). http://dx.doi.org/10.1016/j.polymertesting.2013.07.010

18 Belabed, Z., Houari, M. S. A., Tounsi, A., Beg, A., and Mahmoud, S. R. An efficient and simple higher order shear and normal deformation theory for functionally graded material plates, Composites Part B: Engineering, 60, 274-283, (2014). http://dx.doi.org/10.1016/j.compositesb.2013.12.057

19 Benveniste, Y. A new approach to the application of MoriTanaka's theory in composite materials, Mechanics of Materials, 6, 147-57, (1987). http://dx.doi.org/10.1016/01676636(87)90005-6

20 Mori, T. and Tanaka, K. Average stress in matrix and average elastic energy of materials with mistting inclusions, Acta Metallurgica, 21, 571-574, (1973). http://dx.doi.org/10.1016/0001-6160(73)90064-3

21 Yang, J., Liew, K. M., and Kitipornchai, S. Stochastic analysis of compositionally graded plates with system randomness under static loading, International Journal of Mechanical Science, 47, 1519-41, (2005). http://dx.doi.org/10.1016/j.ijmecsci.2005.06.006
22 Harras, B., Benamar, R., and White, R. G. Experimental and theoretical investigation of the linear and non- linear dynamic behavior of a glare 3 hybrid composite panel, Journal of Sound and Vibration,252 (2), 281-315, (2002). http://dx.doi.org/10.1006/jsvi.2001.3962

$23 \mathrm{Li}$, X. F. A unified approach for analyzing static and dynamic behaviors of functionally graded Timoshenko and Euler-Bernoulli beams, Journal of Sound and Vibration, 318 (4-5), 1210-1229, (2008). http://dx.doi.org/10.1016/j.jsv.2008.04.056

24 Ziane, N., Meftah, S. A., Belhadj, H. A., Tounsi, A., and Bedia, A. A. Free vibration analysis of thin and thick-walled FGB box beams, International Journal of Mechanical Sciences, 66, 273-282, (2013). http://dx.doi.org/10.1016/j.ijmecsci.2012.12.001

25 Meiche, N., Tounsia, A., Zianea, N., Mechaba, I., and Bedia, A. A. A new hyperbolic shear deformation theory for buckling and vibration of functionally graded sandwich plate, International Journal of Mechanical Sciences, 53, 237-247, (2011). http://dx.doi.org/10.1016/j.ijmecsci.2011.01.004

26 Hebali, H., Tounsi, A., Houari, M. S. A., Bessaim, A., and Bedia, A. A. New quasi-3D hyperbolic shear deformation theory for the static and free vibration analysis of functionally graded plates, Journal of Engineering Mechanics, ASCE, 140, 374-383, (2014). http://dx.doi.org/10.1061/(ASCE)EM.1943-7889.0000665

27 Benachour, A., Tahara, H. D., Atmanea, H. A., Tounsia, A., and Ahmeda, M. S. A four variable refined plate theory for free vibrations of functionally graded plates with arbitrary gradient, Composites Part B: Engineering, 42, 1386-1394, (2011). http://dx.doi.org/10.1016/j.compositesb.2011.05.032

28 Meziane, M. A. A., Abdelaziz, H. H., and Tounsi, A. An efficient and simple refined theory for buckling and free vibration of exponentially graded sandwich plates under various boundary conditions, Journal of sandwich structures \& materials, 16 (3), 293-318, (2014). http://dx.doi.org/10.1177/1099636214526852

29 Zidi, M., Tounsi, A., Houari, M. S. A., Bedia, E. A. A., and Beg, O. A. Bending analysis of FGB plates under hygrothermo-mechanical loading using a four variable refined plate theory, Aerospace science and technology, 34, 24-34, (2014). http://dx.doi.org/10.1016/j.ast.2014.02.001

${ }^{30}$ Larbi, L. O., Kaci, A., Houari, M. S. A., and Tounsi, A. An Efficient Shear Deformation Beam Theory Based on Neutral Surface Position for Bending and Free Vibration of Functionally Graded Beams, Mechanics based design of structures and machies, 41 (4), 421-433, (2013). http://dx.doi.org/10.1080/15397734.2013.763713 